\title{
Design and Implementation of Compatible Automatic Transmission Line for Electric Energy Data Acquisition Terminal Testing
}

\author{
Meng Zhang ${ }^{+}$, Gang Kong, Zhibin Fan and Yu Xing \\ State Grid Shandong Electric Power Research Institute, Jinan, Shandong 250003, P. R. China
}

\begin{abstract}
Manual devices are mostly used in the testing of electric energy data acquisition terminals. Under manual testing mode, the handling of acquisition terminals is heavy work and the data interaction and binding process costs a lot of time. In this paper, we present a compatible automatic transmission line for manual terminal testing. The auxiliary line is compatible with various models of acquisition terminal and integrates automatic transmission, automatic identification, information checking, data storage and query function as a whole. The practical application shows improvement in testing efficiency and a great reduction in labor intensity.
\end{abstract}

Keywords: acquisition terminal testing, automatic transmission

\section{Introduction}

With the continuous advance of China's smart grid construction, the demand for intelligent terminal devices in distribution network increases year by year [1]. In order to cope with huge workload of verification and testing, provincial centralized metering centres are established, whose main function is centralized storage, verification, testing and distribution of various electric energy metering related devices such as singleltri-phase energy meter, low voltage current transformer, acquisition terminal, etc.

The automatic verification line systems for energy meters and low voltage current transformers are widely used in these centres in order to augment efficiency and reduce labour intensity. While in the testing of acquisition terminals it is a different case, the manual testing devices are mostly used and only the testing session is auto-completed. The rest sessions such as in/out of warehouse, transportation and handing, data identification and binding, sorting and labelling, are completed manually. Obviously, the adaptation of automatic testing line is most efficiency in time and labour saving. Besides, there are no technical difficulties in realization and are already several applications [2] [3]. There are three main reasons why automatic lines are not widely used in acquisition terminal testing. First, the use of automatic line need more space. For the convenience of automatic transmission and wiring, the testing stations adopt a planar single-layer design, while most manual testing devices have vertical multi-layer structure. It is like the difference of plot ratio between villas and high rise buildings. Apparently the manual testing mode is more space saving. Second, the acquisition terminals have a variety of models and specifications, which varies in size and function [4]. Unlike the uniformity of energy meter [5] and low voltage CT [6], a compatible flexible automatic testing line is necessary, which would be a significant investment compare to the absolute output. Finally, the testing time for a simple terminal takes at least 4 hours. Consider the flat-type single-layer station structure of automatic line, in most time the transmission line is in idle state, the performance of automatic line is not fully utilized. This is like driving a Porsche on a road with a speed limit of $20 \mathrm{~km} / \mathrm{hr}$, which is not efficient economically.

\footnotetext{
+ Corresponding author. Tel.: + 86-18396879529; fax: +86-531-67982700.
}

E-mail address: mengzhangxjtu@126.com. 
Therefore, we present a compatible automatic transmission line in conjunction with manual testing devices, which would undertake most of the workloads except for manual loading/unloading, such as warehouse docking, transmission, turnover box demolition/palletizing, information parsing, sorting, etc. Through the combination of automatic transmission and manual testing, the design idea has the features of less cost, reducing occupied space and higher utilization ratio. At the same time, the labour intensity is reduced and the testing efficiency is augmented.

\section{Transmission Line System Design}

\subsection{Overall Function and Structure}

The main function of the transmission line is to realize the automatic transmission, identification, information parsing and checking of the terminal devices. The transmission line is designed to handle multiple testing tasks issued by the dispatching platform and allocate independent in/out-warehouse cache areas. A brief flow diagram of the transmission line is shown in Fig. 1.

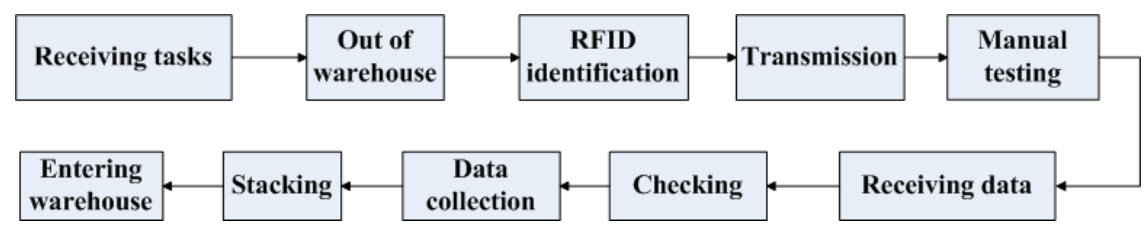

Fig. 1: Brief flow diagram of the transmission line.

The management system applied network control, whose upper and lower system are scheduling platform and manual testing system respectively. Interaction between the three is realized through protocol data interface. The management system obtains tasks from the scheduling platform and allocates them to the testing system, obtains the testing data from it and transfers parsed data to he scheduling platform finally. In addition to data exchange with its upper and lower systems, the management system also has the task allocation, the whole process control, data storage/query, exception handling and other functions. A system architecture diagram is shown in Fig. 2.

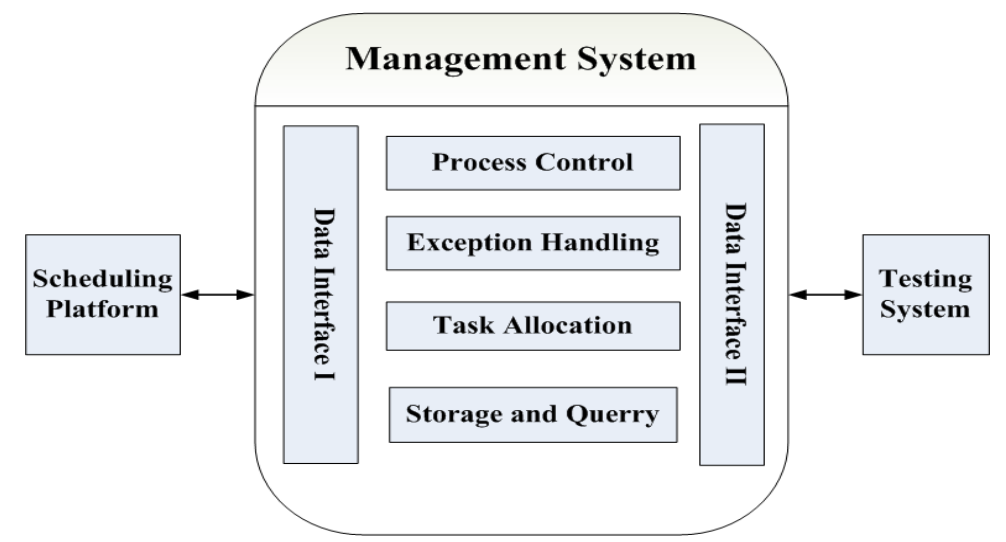

Fig. 2: System architecture of the management system.

\subsection{Functional Unit Structure and Layout}

The functional unit includes a warehouse connection unit, a transmission unit, an information identification unit, and an unstacking/stacking device. The warehouse connection unit mainly enables the connection of the automatic line and the warehouse system so that the turnover box can be transferred accurately between them. The transmission unit can transfer acquisition terminals by single turnover box or by stack ( 5 boxes per stack).Through the roller electrical control technology, the turnover box can be transferred rapidly and reliably between each functional unit.

The information identification unit consists of two parts. One is the out-warehouse identification sub-unit, which can read the whole stack RFID information at one time. The other is the pre-enter warehouse 
identification sub-unit. When the testing process is completed, the bar code label and RFID tags are read by this sub-unit simultaneously, the information is checked before the terminal devices are transferred to the warehouse.

The unstacking/stacking device is in charge of unstacking/stacking, information identification and checking of working boxes. In the end, the turnover box is stored in the warehouse in the form of stacks (5 boxes per stack), which would maximize the use of storage space.

According to the design ideas of each unit, the overall layout of the system is shown as below.

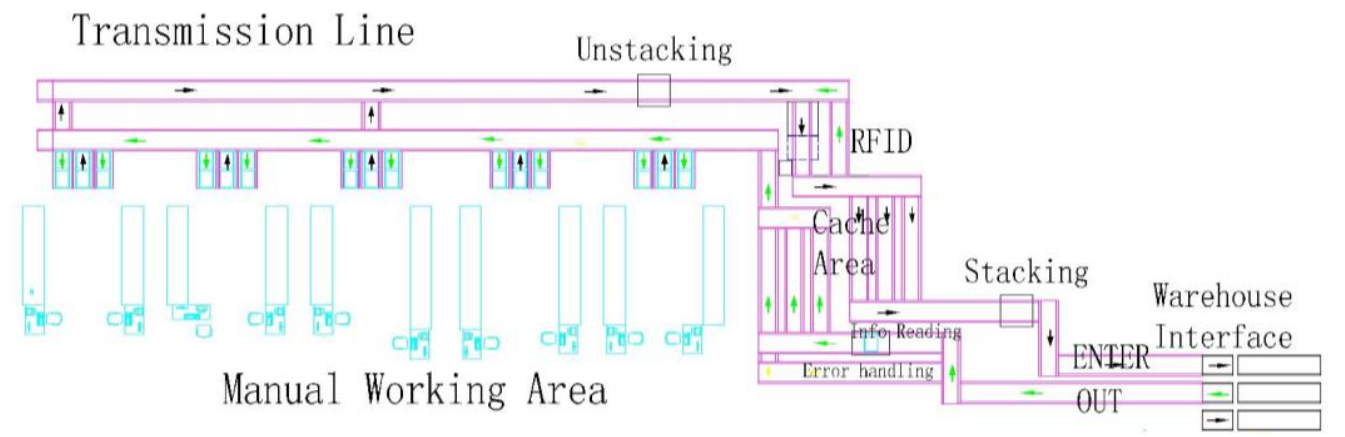

Fig. 3: System layout design.

\subsection{Control System}

The control system consists of a master PLC and a slave PLC station. The master station is responsible for the transmission of the box and information checking. The slave station is responsible for automatic identification and unstacking/stacking. The physical architecture of the control system is shown in Fig. 4.

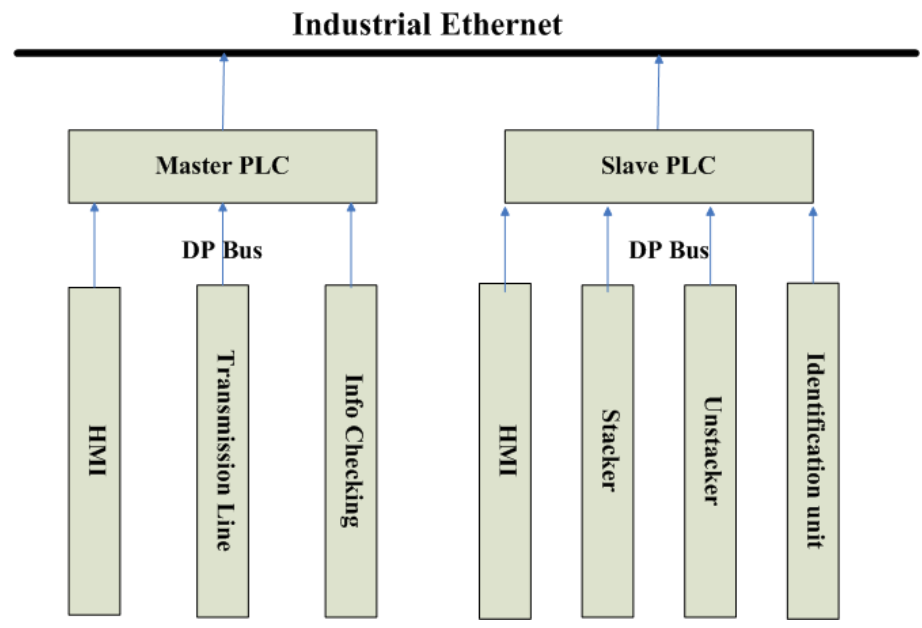

Fig. 4: Physical architecture of the control system.

\subsection{Software System}

The software system consists of the data interface unit, the real-time monitoring unit and the data query unit. Data interface unit receives the mandate issued by the scheduling platform. The real-time monitoring unit transfers the mandate to the control system and monitors the process flow. After the testing is completed, the data is transmitted to the scheduling platform. The data query unit is responsible for the classification, statistics and storage of the data during the whole process.

\subsection{Working Process}

After receipt of tasks from the scheduling platform, the terminal devices are sent to the transmission line in form of stack through the storage connection unit. The devices in stack are processed by the identification unit. If all goes well, they will be assigned to the corresponding buffer area to be tested. Otherwise, they will be transferred to the exceptional area for subsequent processing. After the testing process is complete, the devices in stacks are delivered to the unstacking buffer area, and split into single box through the destacker. 
The bar code and RFID tags information is read and parsed by the identification unit. If the test results are normal and the identity is correct, the boxes will be transferred into the buffer area waiting for palletizing. Otherwise, they will be transferred to the abnormal stacking area for manual processing. Finally, those boxes are stacked again and transferred to the warehouse interface. The complete workflow is shown in Figure 5.

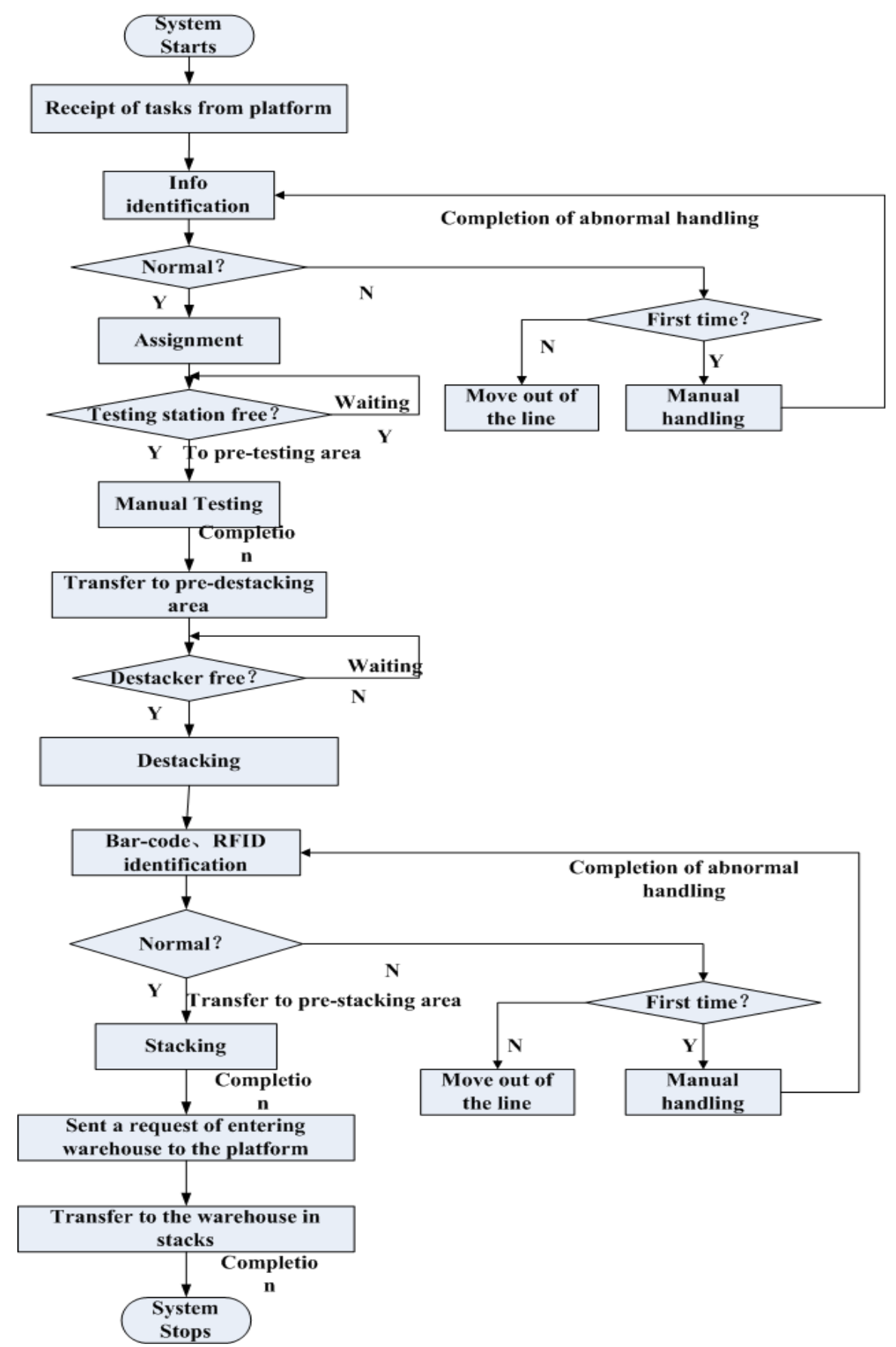

Fig. 5: Workflow of the transmission line system.

\section{Application Results Analysis and Conclusions}

At present, the auxiliary transmission line system has been successfully put into operation in Shandong Electric Energy Metering Centre. The system can automatically complete the heavy and repetitive tasks such as transportation, sorting, data parsing, information identification and checking, etc, which were all manipulated by manpower previously. Through the integration of automation technology into the manual test mode, the testing output per day is increased by $30 \%$, while the number of related workers decreased by $25 \%$. Compared with the fully automated acquisition testing lines, this design method takes up less space, requires less construction budget, which is worthy of further promotion and applications. 


\section{References}

[1] X. Song, L. Han et al, A Review on Development Practice of Smart Grid Technology in China, Electric Power Construction, 2016, 37 (7), pp. 124-135.

[2] G. Lu, Y. Liu et al, Application of Compatibility Delivery Technique in Acquire Terminal Automatic Testing System, North China Electric Power, 2014,12, pp. 19-23.

[3] Y. Li, C. Cheng, Acquisition Terminal How to Implement Automated Testing, Electrical Measurement \& Instrumentation, 2015, 52 (16A), pp. 139-146.

[4] H. Chen, Y. Zhao, Discussion on Collecting Terminal Test System of Intelligent Power System, 2013, 8, pp.56-59.

[5] Y. Zhang. Research and Application of Intelligent Verification Line System for Electric Energy Meter, Electrical Measurement \& Instrumentation, 2009, 46(12), pp. 74-77.

[6] Z. Deng, X. Ma et al, Research and Application of 0. 4kV Metering Current Transformers Automatic Verification System, Electrical Measurement \& Instrumentation, 2013, 50(573), pp. 95-99. 